\title{
Relación de las Experiencias Sexuales Infanto-Juveniles con la Confianza Diádica y el Temor a la Intimidad, en Estudiantes Universitarios $^{1}$
}

\author{
Relationship of Adolescent Sexual Experiences with Dyadic Trust and Fear of \\ Intimacy in University Students
}

\author{
Raúl E. Martínez Montecinos, Raúl Cevallos Añasco \\ Universidad de Concepción, Chile
}

(Rec: 17 octubre 2008 Acep: 20 noviembre 2008)

\begin{abstract}
Resumen
El objetivo de la investigación fue conocer la asociación entre las experiencias sexuales infanto-juveniles y dos aspectos relevantes en la relación pareja: confianza diádica y temor a la intimidad. Se aplicó a una muestra de 451 estudiantes universitarios, 330 mujeres y 121 hombres, un Cuestionario de Experiencias Sexuales Tempranas y dos escalas Likert para evaluar aspectos de la relación en pareja, específicamente la Dyadic Trust Scale que evalúa confianza diádica y la Fear of Intimacy Scale que mide temor a la intimidad emocional. Las experiencias sexuales infanto-juveniles no mostraron relaciones claras con la confianza diádica o el temor a la intimidad emocional. Sin embargo, la valoración de los efectos percibidos de las experiencias sexuales tempranas, además de asociarse con la voluntariedad y no voluntariedad de tales experiencias, sí mostró relaciones inversas con el temor a la intimidad en mujeres. Se encontró una relación inversa entre confianza diádica y temor a la intimidad. Se analizan estos resultados considerando diferencias por sexo y condición de pareja, contrastándolos con los hallazgos pertinentes de investigaciones previas.

Palabras clave: Experiencias sexuales tempranas, confianza diádica, temor a la intimidad emocional.
\end{abstract}

\begin{abstract}
The objective of the present study was to find a link between early sexual experiences and two important aspects of relationships: fear of intimacy and dyadic trust. An Early Sexual Experience Questionnarie and two Likert Scales, specifically the Dyadic Trust Scale and the Fear of Intimacy Scale, were applied to a sample of 451 college students (121 male, 330 female). As a result it was found that early sexual experiences don't have a clear correlation with dyadic trust or fear of intimacy. However, the value given to the perceived efects of this early sexual experiences, in addition with being asociated with the voluntary and involuntary quality of these experiences, did show an inverse relation with fear of intimacy on women. It found an inverse correlation between dyadic trust and fear of intimacy on men and women. This results are analized, taking into account gender and partner condition differences and contrasting them with pertinent findings of previus studys,
\end{abstract}

Key words: Early sexual experiences, dyadic trust, fear of emotional intimacy.

1 Correspondencia a: Raúl Martínez. Facultad de Ciencias Sociales, Departamento de Psicología, Universidad de Concepción. E mail: ramartin@udec.cl 


\section{Introducción}

Sin duda, la confianza y la intimidad en las relaciones diádicas cercanas son bases firmes para el desarrollo y mantenimiento de una relación de pareja y aportan elementos afectivos que producen bienestar y tranquilidad en sus miembros. Comprensiblemente, desconfiar del otro afectivamente cercano origina conductas defensivas y descompromiso, así como la renuencia a estrechar el vínculo por medio de la intimidad, impide la estabilización y profundización de la relación de pareja.

En el contexto de una relación íntima, la confianza se ha definido como la expectativa de respuesta apropiada de otro a las necesidades personales, en el presente y el futuro (Rempel, Holmes \& Zanna, 1985). Esta confianza no es sólo una estimación cognitiva de la probabilidad de que la pareja actúe acorde a las expectativas, sino también y fundamentalmente, la seguridad emocional garantizada acerca de los motivos y conductas del otro, beneficiosos para sí mismo. Así, Rempel et al. (1985) precisan que la confianza incluye tres componentes: la predictibilidad (derivada de la consistencia conductual de la pareja), la confiabilidad (implica la atribución de una disposición positiva estable en el otro) y la fe, que supone transitar desde la observación de las conductas permanentes y la inferencia de la disposición a ser confiable a la esperanza indudable de que la pareja se preocupara de las necesidades de uno, independientemente de las circunstancias en el presente y el futuro. Larzelere y Huston (1980) definen la confianza como "la extensión en que una persona cree que otra persona (o personas) será benevolente y honesta" (p.595), entendiéndose que la benevolencia está motivada por el interés personal de quien la manifiesta o por el beneficio en conjunto con la pareja (Larzelere \& Huston, 1980).

La intimidad es otro importante elemento en una relación de pareja. Se entiende como un proceso que surge de una secuencia observable de interacciones, en la cual una conducta con riesgo probable de ser castigada por otra persona, es por el contrario, reforzada positivamente (Córdova \& Scott, 2001). Precisan los autores que la intimidad supone compartir estados afectivos desagradables, como el daño sufrido, la pena, etc., y pensamientos de temor, preocupación, fracaso personal, etc., pero también otros positivos como alegría, esperanza, preocupación y amor. El temor a la intimidad es definido por Descutner y Thelen (1991) como "...la capacidad inhibida de un individuo, a causa de la ansiedad, para intercambiar pensamientos y sentimientos de significación personal con otro individuo que es altamente valorado" (p. 219). Este temor alude al contenido, cual es la comunicación personal, a la valencia emocional de ella, es decir, la intensidad de los sentimientos relativos a la información, y a la vulnerabilidad implicada, o sea, la alta estimación por quien recibe lo comunicado. El instrumento elaborado por Descutner y Thelen (1991), aplicado en el presente estudio, mide un constructo semejante al deseo del contacto social inhibido por el temor a la pérdida o al rechazo, y aunque tiene correlación negativa con los de cercanía y confianza en el otro y positiva con temor al abandono, se le puede distinguir de ellos (Thelen, Vander, Muir \& Harmon, 2000).

Por lo dicho, es lógico esperar que tanto la confianza como la disposición a cultivar la intimidad en la adultez, sean adversamente afectados por episodios vitales abusivos. Abundantes estudios y revisiones señalan que los impactos negativos del abuso sexual infantil y adolescente en la salud mental del adulto - a corto, mediano y largo plazo - se traducen en diversas manifestaciones psicopatológicas tales como depresión, ansiedad, estrés post-traumático, intensa irritabilidad crónica, abuso de sustancias químicas y adicciones, trastornos alimentarios, proclividad al suicidio, etc. (Briere \& Elliot, 1994; Briere \& Elliot, 2003; Briere \& Runtz, 1993; Coxell, King, Mezey \& Gordon, 1999; Kendall-Tackett, Williams \& Finkelhorn, 1993; King, Coxell \& Mezey, 2002; Molnar, Buka \& Kessler,2001; Spataro, Mullen, Burgess, Wells \& Moss, 2004). Otras investigaciones comunican consecuencias adversas en el ámbito interpersonal. En términos generales, las víctimas del abuso sexual infantil tienen menos amigos, menor cercanía a los padres, pobre ajuste social, sentimientos de aislamiento, temor y desconfianza de otros, dificultad para formar y mantener relaciones íntimas, establecimiento de relaciones abusivas y dificultades en la intimidad sexual (Briere \& Runtz, 1993). Hay datos que permiten afirmar que una historia de abuso sexual se asocia en la adultez con apegos inseguros y desorganizados en la relación de pareja (Mullen \& Fleming, 1998). Mujeres estudiantes de college sexualmente abusadas, informan más baja satisfacción en sus relaciones comprometidas, en comparación con las no abusadas (DiLillo\& Long, 1999), igual que en una muestra de población general autoseleccionada (Hunter, 1991) y en 248 mujeres de una muestra al azar de 2.250 mujeres en las que el abuso se asoció particularmente a inestabilidad de sus relaciones íntimas, baja satisfacción en sus relaciones heterosexuales por su renuencia a discutir asuntos personales con sus parejas, además de percibir un alto control intrusivo y baja preocupación de parte de ellas. Más específicamente, se sintieron capaces de confiar problemas personales a su pareja el $45.6 \%$ de las abusadas y el $62.7 \%$ de las controles, mientras que el $23 \%$ de las primeras informó no tener una comunicación íntima significativa con su pareja, en comparación con el 6\% de las segundas (Mullen, Martin, Anderson, Romans \& Herbison, 1994). Aunque en este último estudio la confianza fue evaluada con una sola pregunta en la entrevista, otros autores que han usado medidas más apropiadas indican también que las mujeres abusadas tienen una significativa más pobre comunicación, más bajo nivel de confianza en sus parejas y menor satisfacción con una relación de al menos seis meses de duración en comparación con las no abusadas, diferencia mantenida luego de controlar 
factores demográficos como estado civil, edad, condición socio-económica y raza (DiLillo \& Long, 1999).

Algunos de los factores que se asocian a una más probable y mayor perturbación derivada del abuso sexual son la temprana edad de ocurrencia, su frecuencia y extensión temporal, el uso de la fuerza, el mayor número de abusadores y su condición incestuosa (Briere \& Elliot, 1994). Briere y Elliot (2003) señalan que las características del abuso sexual específicamente asociadas a 10 síntomas psicológicos que evaluaron fueron la mayor edad al momento de sufrir el abuso, mayor número de incidentes, múltiples abusadores, la penetración oral, anal o vaginal y el mayor nivel de conmoción emocional al momento del abuso, destacándose la no diferencia del efecto de los abusos intra y extra familiar.

Puesto que los estudios más antiguos en relación al abuso sexual fueron llevados a cabo en muestras clínicas, compuestas casi exclusivamente por los niños más severamente abusados, era muy probable constatar la estrecha relación de tal experiencia con efectos adversos. Investigaciones posteriores, de corte más bien epidemiológico (examinando el rango total de las experiencias de abuso sexual y de otro tipo) informan de efectos variados e incluso de ninguno en particular (Mullen \& Fleming, 1998). Hasta un cuarto de los niños abusados sexualmente no comunicaron o no manifestaron la sintomatología que debiera esperarse dentro de 2 años desde el abuso, aunque algunos de éstos pudieron presentar más problemas a los 18 meses que los niños inicialmente afectados de modo evidente (Briere \& Elliot, 1994). En su estudio de 935 hombres y mujeres de una muestra al azar, geográficamente estratificada, Briere y Elliot (2003) precisan que aunque la relación entre abuso infantil (sexual y físico) y una variedad de síntomas psicológicos es estadísticamente robusta, el tamaño de la relación abuso-síntomas es relativamente pequeña. De allí se sigue que el impacto del abuso puede variar de persona a persona en función de otras variables y que no siempre produce efectos a largo plazo, ni que todos los efectos son inevitablemente importantes.

La coexistencia de los maltratos físico y psicológico/ emocional, el abandono, la disfuncionalidad familiar, la observación de violencia en el hogar, la ruptura del vínculo matrimonial, entre otros aspectos del desarrollo temprano, ha hecho pensar que la relación entre abuso sexual infantil y las manifestaciones psicopatológicas individuales, así como las perturbaciones de las relaciones interpersonales se explica en gran medida por estas terceras variables (Briere \& Elliot, 1994; Higgins 2004; Mullen et al., 1994, Mullen \& Fleming, 1998; Rind, Tromovitch \& Bauserman, 1998). Rind y Tromovitch (1997) critican varias revisiones anteriores a su metaanálisis acerca de los efectos del abuso sexual temprano, por los sesgos de muestreo en cuanto los estudios analizados incluyeron muestras clínicas o legales no representativas de la población general, objetando también su imprecisión y subjetividad, pues a excepción de dos, la mayoría de las revisiones habían sido cualitativas. Los autores revisaron siete estudios de muestras nacionales al azar seleccionadas de la población general. Cuatro de los siete estudios provinieron de los Estados Unidos y uno de Gran Bretaña, Canadá y España, respectivamente, concluyendo que sólo una pequeña proporción de personas con experiencia de abuso sexual permanecen dañados y que dentro de ese número una porción substancialmente mayor de mujeres que de hombres sufre un efecto negativo; señalando además que aunque hombres y mujeres que sufren de abuso presentan un ajuste psicológico más pobre que los controles, la magnitud de la relación es pequeña y que por aparecer sólo cuando no se controlan apropiadamente terceras variables, el efecto causal del abuso sexual temprano no puede asegurarse. Cuando se han controlado algunas de ellas, los tamaños de efecto son más bien bajos, de tal manera que la relación causal descubierta inicialmente resulta ser menor y aún incierta (Rind et al., 1998). En su metaanálisis, Rind et al. (1998) los codificaron los resultados de 59 estudios con muestras de estudiantes de college, hombres y mujeres, en 18 categorías de correlatos psicológicos del abuso, entre ellos la susceptibilidad interpersonal (dificultad y marcada incomodidad al interactuar con otros, sentimientos de inadecuación e inferioridad personal) y ajuste social. Al examinar la intensidad de los efectos o correlatos psicológicos de 54 muestras que pudieron ser sometidas a cómputo, el tamaño de efecto estimado, teniendo en cuenta 15.912 participantes fue de $r=.09$, valor que indica que los estudiantes abusados fueron levemente menos ajustados psicológicamente en general que los controles. Los valores $r$ de tamaño del efecto fueron de .10 y .07 , para susceptibilidad interpersonal y ajuste social, respectivamente, ambos claramente pequeños. Al examinar la relación entre ambiente familiar y síntomas, concluyen que en la población de college el ambiente familiar negativo es un factor más importante que el abuso sexual; específicamente, para sensibilidad interpersonal el $\mathrm{r}$ es de $.32 \mathrm{y}$ para el ajuste social $\mathrm{r}=.41$, valores medio a alto.

En el presente estudio se reemplazó la denominación habitual de "abuso sexual infantil" por "experiencia sexual infanto-juvenil no voluntaria". Esta modificación conceptual es sustentada por diversas consideraciones, destacando entre ellas las de Rind et al. (1998), quienes, a partir de los resultados de su meta-análisis, estiman conveniente reexaminar la validez científica de los términos tradicionales utilizados por la mayoría de los autores. Rind et al. (1998) argumentan que clasificar una conducta como abusiva porque es generalmente juzgada inmoral o ilegal, puede ocultar su real naturaleza y sus verdaderas causas y efectos. Como ya se expresó (Martínez, Cevallos \& Reyes, 2008), puesto que en la perspectiva psicológica es esencial el interés por prevenir y reparar los daños sufridos eventualmente por quien ha sido esencialmente receptor de una actividad 
sexual ocurrida tempranamente en su vida, debe tomarse en cuenta el grado de la posible voluntariedad del menor en la participación, así como su evaluación subjetiva del hecho. El estudio -parte de uno de mayor amplitud- tuvo como objetivo examinar la relación entre las experiencias sexuales infanto-juveniles (ESIJ) tanto voluntarias (V) como no voluntarias (noV), categorizadas según su nivel de intimidad, ocurridas dentro de tres rangos de edad, y aspectos de la relación en pareja como son la confianza diádica y la actitud frente a la intimidad en hombres y mujeres estudiantes universitarios. Aunque varios hallazgos indican que mujeres y hombres sexualmente abusados siendo menores, experimentan escasa confianza interpersonal y dificultades para cultivar la intimidad en las relaciones de pareja, otro número de datos pone en duda la constancia de este efecto. La explicación de las contradicciones podría encontrarse en las diferencias metodológicas entre los muchos estudios y revisiones analizados, en cuanto a la definición de abuso sexual, el tipo de muestra utilizado, los medios de recolección de la información, etc. (Goldman \& Padayachi, 2000) además de la interpretación de los resultados (Rind et al. (1998). Este hecho impidió plantear hipótesis que tuviesen un claro e inequívoco respaldo empírico.

\section{Método}

\section{Participantes}

Se eligieron 451 estudiantes de 22 cursos de 16 diferentes carreras de la Universidad de Concepción (Chile), 121 hombres $(26,8 \%)$ y 330 mujeres (73.2\%). La edad media de la muestra fue 20.4 años con D.E. de 2.18 y rango entre 18 y 36 años. El $41.7 \%$ de la muestra tenía una relación sentimental matrimonial, de convivencia o noviazgo y el $58.3 \%$ no tenía pareja.

\section{Instrumentos}

El instrumento utilizado tiene varias secciones, señalándose aquí sólo las pertinentes para el presente estudio. El Cuestionario de Experiencias Sexuales Tempranas (CEST), elaborado sobre la base de las preguntas para evaluar abuso sexual infantil utilizadas por Najman, Dunne, Purdie, Boyle y Coxeter (2005) y por Swahnberg y Wijma (2003), contiene 10 enunciados específicos y breves que aluden a conductas sexuales interpersonales (Martínez et al., 2008). Considerando que los sujetos podrían haber vivido ESIJs en más de una oportunidad y circunstancia, el CEST se construyó de tal manera que un mismo respondiente marcara sus experiencias en más de una categoría (rangos de edad, voluntariedad-no voluntariedad).

Para evaluar las variables de relación interpersonal íntima,se utilizaron la Escala de Confianza Diádica (The Dyadic Trust Scale) de Larzelere y Huston (1980) y la
Escala de Temor a la Intimidad (Fear of Intimacy Scale) de Descutner y Thelen (1991). La primera contiene 8 afirmaciones que deben suscribirse utilizando una escala de 7 niveles entre Fuerte Desacuerdo y Fuerte Acuerdo. Items de ejemplo son: "Hay ocasiones en que no puedo confiar en mi pareja", "Mi pareja es absolutamente honesta y veraz conmigo", "Siento que no puedo contar con mi pareja para que me ayude". El valor más bajo posible de la escala es de 0 y 8 para los sujetos sin y con pareja, respectivamente, mientras que el máximo para ambos grupos corresponde a 56. La segunda escala está conformada originalmente por 35 afirmaciones, de las cuales se eligieron 15 , debiendo el respondiente indicar el grado de su ajuste personal a ellas en una escala con 5 niveles de respuesta a la pregunta ¿cuánto se aplica a mí? (No, en absoluto, Ligeramente, Moderadamente, Bastante y Sí, absolutamente), lo cual resulta en valores posibles mínimo y máximo de 15 y 75 , respectivamente. Se optó por reducir el número de ítems, atendiendo a la cantidad de información solicitada en el estudio total, considerando por otra parte que la consistencia interna de la escala original (coeficiente alfa de .93) permitía utilizar una porción del instrumento total, sin perder la información deseada. Algunos de los ítems son: "Me sentiría incómodo contándole a mi pareja cosas de mi pasado que me han hecho sentir vergüenza", "Me sentiría probablemente nervioso si mostrara a mi pareja fuertes sentimientos de afecto", "A veces me sentiría incómodo escuchando los problemas de mi pareja", "Me sentiría relajado conversando con mi pareja acerca de nuestras metas u objetivos personales". Los alfa de Cronbach calculados para la Escala de Confianza Diádica y los 15 ítems escogidos de la Escala de Temor a la Intimidad fueron de .842 y .664, respectivamente.

\section{Procedimiento}

Se indican las actividades de los respondientes, considerando sólo lo pertinente para el presente estudio. En primer lugar se solicitaron datos demográficos del respondiente, específicamente la edad, el sexo y el estado civil (casado/ conviviente, en relación de pareja sentimental o sin relación de pareja). El Cuestionario fue autoadministrado y anónimo. Para cada ítem del CEST el/la respondiente debió anotar una $\mathrm{X}$ indicando en primer lugar el/los rango/s de edad en que ocurrió la experiencia (3-7, 8-12 años y 13-17 años), o bien, marcar No realizado. Luego, debió señalar si había participado en la acción, voluntaria o no voluntariamente, para finalmente valorar el efecto de la/s experiencia/s en él/ ella al momento presente, considerando 5 conceptos (muy negativo $=1$, negativo $=2$, indiferente $=3$, positivo $=4 \mathrm{y}$ muy positivo $=5$ ).

El anonimato fue favorecido porque los respondientes sólo debieron hacer marcas de selección de alternativas, sin tener que escribir. El tiempo promedio de respuesta fue de 35 minutos aproximadamente. 


\section{Resultados}

Antes de llevar a cabo los análisis estadísticos utilizando el SPSS, se agruparon los 10 ítems del CEST en 3 niveles de acuerdo al grado de intimidad de las actividades sexuales, a saber: intimidad baja (IB), intimidad moderada (IM) e intimidad alta (IA). Los ítems son los siguientes; de IB (3): "Ver fotos/películas de contenido sexual", "Observar partes íntimas de otro", "Conversar acerca de sexo"; de IM (5): "Ser tocado en regiones genitales", "Tocar a otro los genitales",
"Masturbarse acompañado", "Observar a otro masturbarse", "Otro restriega los genitales contra ti"; de IA (2): "Ser penetrado con objetos"; "Tener relaciones sexuales".

Por el modo de respuesta solicitada en el CEST debe entenderse que aunque se aluda a respondientes, los valores en las Tablas corresponden estrictamente a experiencias informadas y no a sujetos, puesto que un número de éstos pudo informar de más de una de ellas en diferentes circunstancias y condiciones.

Tabla 1. Estadísticos descriptivos de las ESIJ-V y no-V globales, por niveles de intimidad y rango de edad, valoración de las ESIJ, confianza diádica y temor a la intimidad; diferencias entre hombres y mujeres

\begin{tabular}{|c|c|c|c|c|c|c|c|}
\hline & Sexo & $\mathrm{n}$ & Media & D.E. & $\mathrm{t}$ & $\mathrm{gl}$ & $\mathrm{p}$ \\
\hline \multirow[t]{2}{*}{ ESIJ-V globales } & Hombre & 121 & 5.27 & 2.37 & 5.44 & 232.96 & .001 \\
\hline & Mujer & 330 & 3.89 & 2.61 & & & \\
\hline \multirow[t]{2}{*}{ ESIJ-V de IB } & Hombre & 121 & 2.58 & 0.69 & 8.07 & 261.07 & .001 \\
\hline & Mujer & 330 & 1.95 & 0.85 & & & \\
\hline \multirow[t]{2}{*}{ ESIJ-V de IM } & Hombre & 121 & 2.16 & 1.63 & 3.55 & 449 & .001 \\
\hline & Mujer & 330 & 1.55 & 1.61 & & & \\
\hline \multirow[t]{2}{*}{ ESIJ-V de IA } & Hombre & 121 & 0.54 & 0.53 & 2.37 & 449 & .018 \\
\hline & Mujer & 330 & 0.39 & 0.58 & & & \\
\hline \multirow[t]{2}{*}{ ESIJ-V, 3-7 años } & Hombre & 121 & 0.21 & 0.73 & 1.99 & 146.22 & .049 \\
\hline & Mujer & 330 & 0.08 & 0.39 & & & \\
\hline \multirow[t]{2}{*}{ ESIJ-V, 8-12 años } & Hombre & 121 & 1.29 & 1.64 & 5.92 & 134.05 & .001 \\
\hline & Mujer & 330 & 0.38 & 0.65 & & & \\
\hline \multirow[t]{2}{*}{ ESIJ-V, 13-17 años } & Hombre & 121 & 4.39 & 2.27 & 3.20 & 236.64 & .002 \\
\hline & Mujer & 330 & 3.59 & 2.54 & & & \\
\hline \multirow[t]{2}{*}{ ESIJ-noV globales } & Hombre & 121 & 0.36 & 0.81 & -0.07 & 449 & .948 \\
\hline & Mujer & 330 & 0.36 & 0.74 & & & \\
\hline \multirow[t]{2}{*}{ ESIJ-noV de IB } & Hombre & 121 & 0.16 & 0.41 & -0.22 & 449 & .830 \\
\hline & Mujer & 330 & 0.17 & 0.43 & & & \\
\hline \multirow[t]{2}{*}{ ESIJ-noV de IM } & Hombre & 121 & 0.19 & 0.47 & -0.02 & 449 & .988 \\
\hline & Mujer & 330 & 0.19 & 0.53 & & & \\
\hline \multirow[t]{2}{*}{ ESIJ-noV de IA } & Hombre & 121 & 0.01 & 0.09 & 0.74 & 449 & ,460 \\
\hline & Mujer & 330 & 0.00 & 0.06 & & & \\
\hline \multirow[t]{2}{*}{ ESIJ-noV, 3-7 años } & Hombre & 121 & 0.04 & 0.37 & 0.07 & 449 & .947 \\
\hline & Mujer & 330 & 0.04 & 0.22 & & & \\
\hline \multirow[t]{2}{*}{ ESIJ-noV, 8-12 años } & Hombre & 121 & 0.14 & 0.43 & -0.26 & 449 & .795 \\
\hline & Mujer & 330 & 0.15 & 0.53 & & & \\
\hline \multirow[t]{2}{*}{ ESIJ-noV, 13-17 años } & Hombre & 121 & 0.19 & 0.51 & 0.34 & 449 & .732 \\
\hline & Mujer & 330 & 0.17 & 0.47 & & & \\
\hline \multirow[t]{2}{*}{$\begin{array}{l}\text { Valoración media de los efectos de } \\
\text { las ESIJ-V y noV }\end{array}$} & Hombre & 118 & 3.95 & 0.63 & 1.11 & 449 & .270 \\
\hline & Mujer & 324 & 3.87 & 0.69 & & & \\
\hline \multirow[t]{2}{*}{ Confianza diádica } & Hombre & 121 & 35.41 & 9.06 & 0.73 & 236.22 & .468 \\
\hline & Mujer & 330 & 34.69 & 10.09 & & & \\
\hline \multirow[t]{2}{*}{ Temor a la intimidad } & Hombre & 121 & 16.08 & 6.94 & 1.57 & 449 & .116 \\
\hline & Mujer & 330 & 14.88 & 7.29 & & & \\
\hline
\end{tabular}


Descripción de variables y diferencias entre grupos

En la Tabla 1 aparecen las medias y D.E. del número de ESIJ-V y noV globales y por niveles de intimidad y rango de edad, de la valoración de las ESIJ, de la confianza diádica y el temor a la intimidad, señalándose además las diferencias entre sexos.

En el grupo de los hombres $(\mathrm{n}=121)$, la media del número de ESIJ-V es de 5.27, sobre un máximo de 9 experiencias del CEST suscritas. La media del número de las ESIJ-noV es bastante más bajo (0.36), con un valor máximo de 4 experiencias. La media de valoración de las ESIJ-V y noV es de 3.95, lo que indica una tendencia hacia la evaluación positiva, teniendo en cuenta además que ningún hombre informó un valor inferior a 2. Las medias para confianza diádica y temor a la intimidad son 35.41 y 16.08 , respectivamente.

Tabla 2. Estadísticos descriptivos de las ESIJ-V y noV globales, por niveles de intimidad y rango de edad, valoración de las ESIJ, confianza diádica y temor a la intimidad; diferencias por condición de la relación en pareja

\begin{tabular}{|c|c|c|c|c|c|c|c|}
\hline & $\begin{array}{l}\text { Condición de } \\
\text { pareja }\end{array}$ & $\mathrm{n}$ & Media & D.E. & $\mathrm{t}$ & gl & $\mathrm{p}$ \\
\hline \multirow[t]{2}{*}{ ESIJ-V globales } & Sin pareja & 188 & 4.19 & 2.49 & -0.51 & 421.75 & .611 \\
\hline & Con pareja & 263 & 4.31 & 2.71 & & & \\
\hline \multirow[t]{2}{*}{ ESIJ-V de IB } & Sin pareja & 188 & 2.17 & 0.80 & 1.15 & 449 & .251 \\
\hline & Con pareja & 263 & 2.08 & 0.90 & & & \\
\hline \multirow[t]{2}{*}{ ESIJ-V de IM } & Sin pareja & 188 & 1.59 & 1.62 & -1.33 & 449 & .183 \\
\hline & Con pareja & 263 & 1.80 & 1.65 & & & \\
\hline \multirow[t]{2}{*}{ ESIJ-V de IA } & Sin pareja & 188 & 0.43 & 0.56 & -0.22 & 449 & .830 \\
\hline & Con pareja & 263 & 0.44 & 0.58 & & & \\
\hline \multirow[t]{2}{*}{ ESIJ-V, 3-7 años } & Sin pareja & 188 & 0.12 & 0.54 & 0.33 & 449 & .745 \\
\hline & Con pareja & 263 & 0.11 & 0.49 & & & \\
\hline \multirow[t]{2}{*}{ ESIJ-V, 8-12 años } & Sin pareja & 188 & 0.65 & 1.17 & 0.35 & 449 & .724 \\
\hline & Con pareja & 263 & 0.61 & 1.02 & & & \\
\hline \multirow[t]{2}{*}{ ESIJ-V, 13-17 años } & Sin pareja & 188 & 3.68 & 2.32 & -0.91 & 428.22 & .363 \\
\hline & Con pareja & 263 & 3.89 & 2.61 & & & \\
\hline \multirow[t]{2}{*}{ ESIJ-noV globales } & Sin pareja & 188 & 0.38 & 0.72 & 0.57 & 449 & .572 \\
\hline & Con pareja & 263 & 0.34 & 0.78 & & & \\
\hline \multirow[t]{2}{*}{ ESIJ-noV de IB } & Sin pareja & 188 & 0.15 & 0.42 & -0.42 & 449 & .676 \\
\hline & Con pareja & 263 & 0.17 & 0.43 & & & \\
\hline \multirow[t]{2}{*}{ ESIJ-noV de IM } & Sin pareja & 188 & 0.23 & 0.52 & 1.33 & 392.67 & .185 \\
\hline & Con pareja & 263 & 0.16 & 0.50 & & & \\
\hline \multirow[t]{2}{*}{ ESIJ-noV de IA } & Sin pareja & 188 & 0.00 & 0.00 & -1.42 & 262 & .158 \\
\hline & Con pareja & 263 & 0.01 & 0.09 & & & \\
\hline \multirow[t]{2}{*}{ ESIJ-noV, 3-7 años } & Sin pareja & 188 & 0.03 & 0.16 & -0.88 & 449 & .380 \\
\hline & Con pareja & 263 & 0.05 & 0.33 & & & \\
\hline \multirow[t]{2}{*}{ ESIJ-noV, 8-12 años } & Sin pareja & 188 & 0.14 & 0.47 & -0.25 & 449 & .800 \\
\hline & Con pareja & 263 & 0.16 & 0.53 & & & \\
\hline \multirow[t]{2}{*}{ ESIJ-noV, 13-17 años } & Sin pareja & 188 & 0.21 & 0.49 & 1.32 & 386.58 & .187 \\
\hline & Con pareja & 263 & 0.15 & 0.46 & & & \\
\hline \multirow{2}{*}{$\begin{array}{l}\text { Valoración media de los } \\
\text { efectos de } \\
\text { las ESIJ-V y noV }\end{array}$} & Sin pareja & 187 & 3.88 & 0.62 & -0.27 & 449 & .790 \\
\hline & Con pareja & 255 & 3.90 & 0.71 & & & \\
\hline \multirow[t]{2}{*}{ Confianza diádica } & Sin pareja & 188 & 29.46 & 9.17 & -11.22 & 449 & .001 \\
\hline & Con pareja & 263 & 38.76 & 8.33 & & & \\
\hline \multirow[t]{2}{*}{ Temor a la intimidad } & Sin pareja & 188 & 17.72 & 7.22 & 6.55 & 449 & .001 \\
\hline & Con pareja & 263 & 13.40 & 6.65 & & & \\
\hline
\end{tabular}


En la submuestra de mujeres $(\mathrm{n}=330)$, la media del número de ESIJ-V es 3.89, mientras que a las ESIJ-noV corresponde 0.36 , considerando un máximo de 5 experiencias informadas. La media de valoración de las ESIJ-V y noV es de 3.87, levemente inferior a la de los hombres. Los valores promedio para confianza diádica y temor a la intimidad son 34.69 y 14.88 , respectivamente.

Entre ambos grupos (hombres y mujeres) se encontraron diferencias significativas mediante prueba $t$, en el número global de las ESIJ-V y en todos sus niveles de intimidad y rangos de edad, mientras que no se aprecian respecto a las ESIJ-noV, valoración media del efecto de las ESIJ-V y noV, confianza diádica y temor a la intimidad.

En la Tabla 2 se describen las ESIJ-V y noV globales y por niveles de intimidad y rango de edad, la valoración de las ESIJ, la confianza diádica y el temor a la intimidad, señalándose las diferencias entre hombres y mujeres sin pareja y con pareja.

En el grupo de sujetos (hombres y mujeres) sin pareja ( $\mathrm{n}=188$ ), la media del número global de ESIJ-V es 4.19, con un máximo de 9 de las 10 posibles experiencias. La media de las ESIJ-noV es 0.38 , siendo 5 el número máximo de experiencias informadas. La media de valoración del efecto de las ESIJ-V y noV es 3.88, mientras que para confianza diádica y temor a la intimidad son 29.46 y 17.72, respectivamente. En los sujetos con pareja $(\mathrm{n}=263)$, las medias de las ESIJ-V y las ESIJ-noV son 4.31 y 0.34, respectivamente. La media de valoración de las ESIJ-V y noV es 3.90, cercana al concepto de "positivo". Las medias para confianza diádica y miedo a la intimidad son 38.76 y 13.40 , respectivamente. Entre ambos grupos se encontraron diferencias significativas mediante prueba $t$, sólo en confianza diádica $\mathrm{y}$ temor a la intimidad.

\section{Asociaciones entre variables}

En las Tablas 3 a 6, se indican las asociaciones de las variables de las ESIJ-V y noV por niveles de intimidad y rangos de edad, con confianza diádica y temor a la intimidad, por sexo (Tablas 3 y 5) y por condición de pareja (Tablas 4 y 6).

Tanto en el grupo de hombres como en el de los sujetos con pareja, el número de las ESIJ-V correlaciona positivamente con la valoración media de las ESIJ-Vy noV $(\mathrm{r}=.220$ y $\mathrm{r}=.161$, respectivamente), presentándose dicha relación en ambos casos con más fuerza respecto a las ESIJ-V en el

Tabla 3. Asociaciones entre las ESIJ-V y noV globales, por niveles de intimidad, valoración de las ESIJ y confianza diádica y temor a la intimidad, por sexo

\begin{tabular}{|c|c|c|c|c|}
\hline Variables & Sexo & $\begin{array}{l}\text { Valoración } \\
\text { ESIJ }\end{array}$ & $\begin{array}{r}\text { Confianza } \\
\text { diádica }\end{array}$ & $\begin{array}{c}\text { Temor } \\
\text { intimidad }\end{array}$ \\
\hline \multirow[t]{2}{*}{ ESIJ-V glob. } & M & $.220 *$ & $-.188(*)$ & .054 \\
\hline & $\mathrm{F}$ & .016 & -.002 & -.022 \\
\hline \multirow[t]{2}{*}{ ESIJ-V IB } & M & .146 & -.147 & .075 \\
\hline & $\mathrm{F}$ & -.046 & -.085 & .105 \\
\hline \multirow[t]{2}{*}{ ESIJ-V IM } & M & .171 & -.130 & .039 \\
\hline & $\mathrm{F}$ & .022 & .046 & -.066 \\
\hline \multirow[t]{2}{*}{ ESIJ-V IA } & M & $.257 * *$ & $-.248 * *$ & .022 \\
\hline & $\mathrm{F}$ & .077 & -.011 & -.071 \\
\hline \multirow[t]{2}{*}{ ESIJ-noV glob. } & M & $-.209 *$ & -.019 & .066 \\
\hline & $\mathrm{F}$ & $-.277 * *$ & -.023 & -.024 \\
\hline \multirow[t]{2}{*}{ ESIJ-noV IB } & M & -.113 & -.058 & .119 \\
\hline & $\mathrm{F}$ & $-.135 *$ & .017 & .012 \\
\hline \multirow[t]{2}{*}{ ESIJ-noV IM } & M & $-.242 * *$ & .021 & .010 \\
\hline & $\mathrm{F}$ & $-.267 * *$ & -.046 & -.036 \\
\hline \multirow[t]{2}{*}{ ESIJ-noV IA } & M & -.085 & -.014 & -.001 \\
\hline & $\mathrm{F}$ & -.101 & -.009 & -.075 \\
\hline \multirow[t]{2}{*}{ Valoración media ESIJ } & M & - & -.055 & .083 \\
\hline & $\mathrm{F}$ & - & .081 & $-.151 * *$ \\
\hline \multirow[t]{2}{*}{ Confianza diádica } & M & - & - & $-.399 * *$ \\
\hline & $\mathrm{F}$ & - & - & $-.527 * *$ \\
\hline
\end{tabular}


Tabla 4. Asociaciones entre las ESIJ-V y noV globales, por niveles de intimidad, valoración de las ESIJ y confianza diádica y temor a la intimidad, por condición de pareja

\begin{tabular}{|c|c|c|c|c|}
\hline Variables & $\begin{array}{c}\text { Condición } \\
\text { pareja }\end{array}$ & $\begin{array}{c}\text { Valoración } \\
\text { ESIJ }\end{array}$ & $\begin{array}{l}\text { Confianza } \\
\text { diádica }\end{array}$ & $\begin{array}{l}\text { Temor a la } \\
\text { intimidad }\end{array}$ \\
\hline \multirow[t]{2}{*}{ ESIJ-V glob. } & SP & -.076 & -.088 & .039 \\
\hline & $\mathrm{CP}$ & $.161 *$ & -.028 & .009 \\
\hline \multirow[t]{2}{*}{ ESIJ-V IB } & SP & $-.164 *$ & -.037 & .128 \\
\hline & $\mathrm{CP}$ & .114 & -.085 & .091 \\
\hline \multirow[t]{2}{*}{ ESIJ-V IM } & SP & -.035 & -.077 & -.005 \\
\hline & $\mathrm{CP}$ & $.129 *$ & .017 & -.010 \\
\hline \multirow[t]{2}{*}{ ESIJ-V IA } & SP & -.002 & -.117 & .003 \\
\hline & $\mathrm{CP}$ & $.200 * *$ & -.045 & -.069 \\
\hline \multirow[t]{2}{*}{ ESIJ-noV gl. } & SP & $-.272 * *$ & -.017 & .026 \\
\hline & $\mathrm{CP}$ & $-.250 * *$ & -.007 & -.032 \\
\hline \multirow[t]{2}{*}{ ESIJ-noV IB } & SP & -.129 & .026 & .064 \\
\hline & $\mathrm{CP}$ & $-.132 *$ & -.041 & .032 \\
\hline \multirow[t]{2}{*}{ ESIJ-noV IM } & SP & $-.270 * *$ & -.044 & -.015 \\
\hline & $\mathrm{CP}$ & $-.257 * *$ & .034 & -.070 \\
\hline \multirow[t]{2}{*}{ ESIJ-noV IA } & SP & (a) & (a) & (a) \\
\hline & $\mathrm{CP}$ & -.113 & -.055 & -.038 \\
\hline \multirow[t]{2}{*}{$\begin{array}{l}\text { Valoración } \\
\text { media ESIJ }\end{array}$} & SP & - & .106 & $-.189 * *$ \\
\hline & $\mathrm{CP}$ & - & .013 & -.024 \\
\hline \multirow[t]{2}{*}{ Confianza diádica } & SP & - & - & $-.395 * *$ \\
\hline & $\mathrm{CP}$ & - & - & $-.441 * *$ \\
\hline
\end{tabular}

Nota. $\mathrm{SP}=$ Sin pareja $\mathrm{CP}=$ Con pareja

(a) Valor no calculado por muy bajo $\mathrm{n}$ y no variabilidad de datos

$* \mathrm{p}<.05$ (bilateral)

$* * \mathrm{p}<.01$ (bilateral)

Tabla 5. Asociaciones entre las ESIJ-V y noV por rangos de edad, valoración de las ESIJ y confianza diádica y temor a la intimidad, por sexo

\begin{tabular}{|c|c|c|c|c|}
\hline Variables & Sexo & $\begin{array}{l}\text { Valoración } \\
\text { ESIJ }\end{array}$ & $\begin{array}{l}\text { Confianza } \\
\text { diádica }\end{array}$ & $\begin{array}{c}\text { Temor a } \\
\text { intimidad }\end{array}$ \\
\hline \multirow[t]{2}{*}{ ESIJ-V, 3-7 años } & M & -.054 & -.030 & .082 \\
\hline & $\mathrm{F}$ & -.036 & -.002 & .060 \\
\hline \multirow[t]{2}{*}{ ESIJ-V, 8-12 años } & M & .005 & .018 & .094 \\
\hline & $\mathrm{F}$ & -.047 & -.093 & -.007 \\
\hline \multirow[t]{2}{*}{ ESIJ-V, 13-17 años } & M & $.242 * *$ & $-.181 *$ & .025 \\
\hline & $\mathrm{F}$ & .044 & .025 & -.042 \\
\hline \multirow[t]{2}{*}{ ESIJ-noV, 3-7 a. } & M & -.081 & .012 & -.024 \\
\hline & $\mathrm{F}$ & -.035 & -.071 & -.029 \\
\hline \multirow[t]{2}{*}{ ESIJ-noV, 8-12 } & M & $-.206^{*}$ & .061 & .032 \\
\hline & $\mathrm{F}$ & $-.193 * *$ & .026 & -.077 \\
\hline \multirow[t]{2}{*}{ ESIJ-noV, 13-17 } & M & -.091 & -.059 & .055 \\
\hline & $\mathrm{F}$ & $-.199 * *$ & -.021 & .060 \\
\hline
\end{tabular}

\footnotetext{
$* \mathrm{p}<.05$ (bilateral)

$* * \mathrm{p}<.01$ (bilateral)
} 
Tabla 6. Asociaciones entre las ESIJ-V y noV por rangos de edad, valoración de las ESIJ y confianza diádica y temor a la intimidad, por condición de pareja

\begin{tabular}{lcccc}
\hline Variables & $\begin{array}{c}\text { Condic. } \\
\text { Pareja }\end{array}$ & $\begin{array}{c}\text { Valoración } \\
\text { ESIJ }\end{array}$ & $\begin{array}{c}\text { Confianza } \\
\text { diádica }\end{array}$ & $\begin{array}{c}\text { Temor a } \\
\text { intimidad }\end{array}$ \\
\hline ESIJ-V, 3-7 años & $\mathrm{SP}$ & .004 & .018 & .050 \\
& $\mathrm{CP}$ & -.059 & -.016 & .091 \\
ESIJ-V, 8-12 años & $\mathrm{SP}$ & -.017 & .036 & .039 \\
& $\mathrm{CP}$ & .014 & -.065 & .075 \\
ESIJ-V,13-17 años & $\mathrm{SP}$ & -.060 & -.079 & .023 \\
& $\mathrm{CP}$ & $.183 * *$ & -.016 & -.021 \\
ESIJ-noV, 3-7 años & $\mathrm{SP}$ & -.020 & -.117 & -.053 \\
\multirow{2}{*}{ ESIJ-noV, 8-12 a. } & $\mathrm{CP}$ & -.061 & -.054 & .000 \\
& $\mathrm{SP}$ & $-.198 * *$ & .117 & -057 \\
ESIJ-noV, 13-17 a. & $\mathrm{CP}$ & $-.194 * *$ & -.027 & -.050 \\
& $\mathrm{SP}$ & $-.200 * *$ & -.097 & .110 \\
& $\mathrm{CP}$ & $-.148 *$ & .080 & -.013 \\
\hline
\end{tabular}

$* \mathrm{p}<.05$ (bilateral)

** $\mathrm{p}<.01$ (bilateral)

rango de 13 a 17 años (hombres $r=.242$; sujetos con pareja $\mathrm{r}=.183$ ) y para las ESIJ-V de IA (hombres $\mathrm{r}=.257$; sujetos con pareja $\mathrm{r}=.200)$ El número de las ESIJ-noV correlaciona negativamente con la valoración media de las $\mathrm{ESIJ}-\mathrm{V}$ y noV, en los cuatro grupos estudiados (hombres: $\mathrm{r}=-.209$; mujeres $\mathrm{r}=-.277$; sujetos $\sin$ pareja: $\mathrm{r}=-.272$; sujetos con pareja: $\mathrm{r}$ $=-.250$ ), relación que aparece fundamentalmente respecto a las ESIJ-noV ocurridas en el segundo y tercer rango de edad (hombres, 8-12 años: $r=-.206$; mujeres, 8-12 años: $r=$ -.193 , y 13-17 años: $r=-.199$; sujetos sin pareja, 8-12 años: $\mathrm{r}=-.198$, y 13-17 años: $\mathrm{r}=-.200$; sujetos con pareja, 8-12 años: $\mathrm{r}=-.194$, y $13-17$ años: $\mathrm{r}=-.148$ ) y de IM (hombres: $\mathrm{r}=$ -.242 ; mujeres: $\mathrm{r}=-.267$; sujetos sin pareja: $\mathrm{r}=-.270$; sujetos con pareja: $\mathrm{r}=-.257$ ). En el grupo de hombres, se presenta una correlación negativa entre el número de las ESIJ-V y la confianza diádica $(\mathrm{r}=-.188)$, relación que asume mayor fuerza respecto a las ESIJ-V en el rango entre 13 y 17 años $(\mathrm{r}=-.181)$ y de IA $(\mathrm{r}=-.248)$. Además, tanto en el grupo de mujeres como en el de los sujetos sin pareja, la valoración de las ESIJ-Vy noV correlaciona negativamente con el temor a la intimidad, con $\mathrm{r}=-.151$ y $\mathrm{r}=-.189$, respectivamente. La confianza diádica correlaciona negativamente con el temor a la intimidad en los cuatro grupos estudiados (hombres: $r=$ -.399 ; mujeres: $\mathrm{r}=-.527$; sujetos sin pareja: $\mathrm{r}=-.395$; sujetos con pareja: $\mathrm{r}=-.441$ ).

\section{Discusión}

En los hombres y los sujetos (hombres y mujeres) con pareja aparecen asociados en forma directa el número de ESIJ-V vividas y la valoración del efecto de las ESIJ-V y noV en conjunto. Esta relación se puede comprender sin dificultad, pues la voluntariedad de participación en una actividad íntima sexual implica una disposición positiva que aumenta la probabilidad de percibir consecuencias en el mismo sentido, las que pesan más por ser mayor el número de ESIJ-V que el de ESIJ-noV. Por una razón semejante es comprensible la relación inversa entre el número de ESIJ-noV y la valoración media de las ESIJ consideradas globalmente, en hombres y mujeres, así como en sujetos sin y con pareja.

Por el contrario, no es explicable por qué en las mujeres la correlación positiva entre el número de ESIJ-V y la valoración de las ESIJ-V y noV no alcanza significación, así como que en los sujetos sin pareja la correlación es negativa aunque no significativa.

La relación inversa entre el número de ESIJ-V globales y la confianza diádica en hombres, deriva del mayor peso negativo de las ESIJ-V de IA, pues las de IB e IM aparecen con una correlación positiva aunque no significativa. Una explicación especulativa aludiría a que los hombres llegan a desconfiar genéricamente de la mujer en la medida que su mayor número de experiencias coitales (ESIJ de IA), probablemente con un número de mujeres diferentes, les hace suponer que ellas podrían ser fácilmente influidas para llegar a la máxima intimidad sexual, a costa de una posible infidelidad.

El número de ESIJ-V no aporta significativamente al parecer en ningún sentido a la confianza diádica y al temor a la intimidad de las mujeres.

La asociación entre mayor (mejor) valoración de los efectos de las ESIJ-V y noV y el menor temor a la intimidad emocional en las mujeres es lógica y psicológicamente comprensible. La correlación positiva pero no significativa 
en hombres, indicaría cierta independencia del desarrollo de la intimidad emocional en ellos, al margen de las ESIJ, mientras que en las mujeres pareciera ser que las experiencias sexuales percibidas más bien positivamente y la intimidad emocional están estrechamente ligadas.

Existe una muy significativa relación inversa entre la confianza diádica y el temor a la intimidad emocional, independientemente del sexo y la condición de pareja de los respondientes. Este dato, lógicamente comprensible, se orienta además en la línea del hallazgo de Descutner y Thelen (1991), quienes en el proceso de validar la Escala de Temor a la Intimidad en una muestra universitaria, encontraron una correlación negativa entre este constructo y la autorrevelación $(\mathrm{r}=-.52)$, así como con los datos de Doi y Thelen (1993) que en una muestra de 83 hombres y 88 mujeres entre 35 y 55 años de edad, hallaron una correlación negativa con confianza en el otro $(\mathrm{r}=-.40)$.

El número de las ESIJ-noV globales, por niveles de intimidad y rangos de edad, no mostró asociación con las variables de relación de pareja escogidas para este estudio, en ninguna de las dos agrupaciones de los sujetos (por sexo y condición de pareja), lo cual permite suponer que la no voluntariedad de las experiencias sexuales tempranas no necesariamente genera consecuencias negativas para condiciones psicológicas como la confianza diádica y la actitud positiva ante la intimidad, que son bases del establecimiento y mantenimiento de una relación de pareja. La independencia de hechos sexuales tempranos no deseados y diversos posibles resultados -entre ellos algunos procesos que facilitan la vinculación diádica- ha sido señalada y demostrada por dos metaanálisis de grupos no clínicos ya comentados en la introducción; uno que analizó estudios de muestras nacionales de diversos países (Rind \& Tromovitch,1997) y otro que consideró muestras de estudiantes de college (Rind et al.,1998). Tales hallazgos son consistentes con los resultados de KendallTackett et al. (1993), quienes revisaron 45 estudios acerca del efecto del abuso sexual infantil en muestras extraídas principalmente de programas de evaluación y tratamiento psicológico. Los autores encontraron que más del $49 \%$ de los niños que sufrieron la experiencia no mostraba daño psicológico, consignando además que, en la mayoría de los niños perturbados, los síntomas desaparecieron dentro de dos años con o sin tratamiento. Una interesante línea de investigación indica que el mayor impacto negativo sobre aspectos personales y de relación interpersonal deriva más claramente de la coexistencia de diversos maltratos no sexuales y de las disfunciones familiares que de las experiencias sexuales abusivas per se (Briere \& Elliot, 1994; Higgins, 2004; Mullen et al., 1994, Mullen \& Fleming, 1998). Esta evidencia induciría a suponer que, en general, en la vida de los hombres y mujeres del presente estudio estuvieron ausentes tales elementos adicionales. A la luz de los hallazgos de este estudio y los datos procedentes de otras investigaciones, parece apropiada la sugerencia de Higgins (2004) en cuanto a no investigar los efectos de los diversos tipos de abuso o maltrato temprano por separado, sino más bien establecer niveles por sumatoria de maltratos con independencia de sus tipos, pues todos ellos afectarían procesos psicológicos comunes, con resultados semejantes (Edwards, Holden, Felitti \& Anda, 2003).

Por otra parte, teniendo en mente la relación inversa descubierta en este estudio entre las ESIJ-noV y la valoración de sus efectos percibidos, una explicación alternativa a la ausencia de la asociación esperable entre ESIJ-noV y las variables de relación de pareja (confianza diádica y temor a la intimidad), implicaría suponer que aquellos que han vivido este tipo de experiencias han sido capaces de redefinirlas y sobreponerse a las eventuales consecuencias negativas, presentando en la adolescencia tardía y adultez, características similares en aspectos pertinentes a la relación de pareja, a quienes no las han vivido. Este razonamiento orienta hacia la necesidad de investigar las capacidades de resistencia psicológica al estrés derivado de circunstancias sexuales tempranas no deseadas y aquéllas que permiten la recuperación de un eventual daño. Como forma de lograr un conocimiento más completo, apropiado y útil, DiLillo (2001) sugiere que las investigaciones no sólo evalúen el abuso sexual infantil sino también la exposición a fuentes potenciales de trauma tales como desastres naturales, procedimientos médicos estresantes, accidentes, etc., de tal forma que aislando estas variables se pueda medir el efecto neto de dicha experiencia íntima.

En definitiva, en esta investigación con estudiantes universitarios, se descubrió un número de asociaciones positivas y negativas (estadísticamente significativas) entre las variables de las ESIJ, la valoración de ellas y dos condiciones de la relación en pareja. Algunas de ellas son comprensibles, otras además apoyadas por hallazgos de estudios anteriores, y por último, un número difícil de explicar, respecto a las cuales cabe indagar en una futura investigación algún fenómeno mediador que no fue considerado en esta ocasión. Obviamente, para superar la limitación de este estudio en cuanto a usar una muestra de estudiantes universitarios exclusivamente, sería deseable una investigación semejante con otras poblaciones no clínicas de hombres y mujeres, de edad similar y también mayor, pues es posible que los estudiantes universitarios sean sólo representativos de una población de cierto nivel educacional, quizás con mayores competencias psicológicas que les faciliten la recuperación de eventos vitales estresantes y/o que sean aún demasiado jóvenes como para manifestar un efecto adverso. De todos modos, es importante destacar la utilidad del conocimiento obtenido en nuestro medio, aplicable a un creciente porcentaje de nuestra población general no clínica, al mismo tiempo que recordar la imposibilidad de establecer relaciones causales a partir de una investigación correlacional. 
A partir de los resultados del estudio y la revisión bibliográfica efectuada, parece conveniente asumir una posición de mayor ponderación y equilibrio respecto a las eventuales consecuencias del denominado "abuso sexual infantil". No es posible afirmar sin reservas que las ESIJ-noV, de diferentes niveles de intimidad, con pares o adultos, acontecidas entre los 3 y 17 años de edad, aunque valoradas negativamente de modo subjetivo, sean siempre dañinas para todas las personas, y que sus posibles efectos adversos sean deletéreos e irreversibles. Esta constatación debiera ser una muy buena noticia para los padres, profesionales y adultos en general que temen la peor consecuencia para los niños, preadolescentes y adolescentes, y obviamente con mayor razón para los directamente comprometidos. Junto a esta mirada de mayor mesura del fenómeno que nos ocupa, es éticamente obligatorio seguir prestando la mayor atención y dedicando los más amplios recursos a la prevención del daño y la recuperación de quienes resulten verdaderamente afectados por una experiencia sexual abusiva.

\section{Referencias}

Briere, J. \& Elliot. D.M. (1994). Immediate and long-term impacts of child sexual abuse. The Future of Children, 4 (2), 54-69.

Briere, J. \& Runtz, M. (1993). Childhood sexual abuse: Long-term sequelae and implications for psychological assessment. Journal of Interpersonal Violence, 8 (3), 312-330.

Briere, J. \& Elliot, D.M. (2003). Prevalence and psychological sequelae of self-reported childhood physical and sexual abuse in a general population simple of men and women. Child Abuse and Neglect, 27, 1205-1222.

Córdova, J.V. \& Scott, R.L. (2001). Intimacy: A behavioral interpretation. The Behavior Analyst, 24 (1), 75-86.

Coxell, A., King, M., Mezey, G. \& Gordon, D. (1999). Lifetime prevalence, characteristics, and associated problems of non-consensual sex in men: cross sectional survey. British Medical Journal, 318, 846-850.

Descutner, C.J. \& Thelen, M.H. (1991) Development and validation of a Fear-of Intimacy Scale. Psychological Assessment: A Journal of Consulting and Clinical Psychology, 3, (2), 218-225.

DiLillo, D. (2001). Interpersonal functioning among women reporting a history of childhood sexual abuse: Empirical findings and methodological isues. Clinical Psychology Review, 21, 553-576.

DiLillo, D. \& Long, P.J. ( 1999). Perceptions of couple functioning among female survivors of child sexual abuse. Journal of Child Sexual Abuse, 7, 59-76.

Doi, S.C. \& Thelen, M.H. (1993). The Fear of Intimacy Scale: Replication and extension. Psychological Assesment, 5 (3), 377-383.

Edwards, V.J., Holden, G.W., Felitti, V.J. \& Anda, R.F. (2003). Relationship between multiple forms of childhood maltreatment and adult mental health in community respondents: Results from the adverse childhood experiences study. American Journal of Psychiatry, 160, 1453-1460.
Goldman, J.D.G. \& Padayachi, U. K. (2000). Some methodological problems in estimating incidence and prevalence in child sexual abuse research. The Journal of Sex Research, 37 (4), 305-314.

Higgins, D. (2004). Differentiating between child maltreatment experiences. Australian Institute of Family Studies. Family Matters $\mathrm{N}^{\mathbf{0}} 69$, Spring/Summer. Recuperado el 7 de julio de 2008 desde http://www. aifs.gov.au/institute.

Hunter, J.A. (1991). A comparison of the psychosocial maladjustment of adult males and females sexually abused as children. Journal of Interpersonal Violence, 6, 205-217.

Kendall-Tackett, K.A., Williams, L.M. \& Finkelhorn, D. (1993). Impact of sexual abuse on children: A review and synthesis of recent empirical studies. Psychological Bulletin, 113 (1), 164-180.

King, M., Coxell, A. \& Mezey, G. (2002). Sexual molestation of males: associations with psychological disturbance. British Journal of Psychiatry, 181, 153-157.

Larzelere, R.E. \& Huston, T.L. (1980). The Dyadic Trust Scale: Toward understanding interpersonal trust in close relationships. Journal of Marriage and the Family, 42 (3), 595-604.

Martínez, R.E., Cevallos, R. \& Reyes, F. (2008). Experiencias sexuales infanto-juveniles voluntarias y no voluntarias, con pares y adultos y efectos percibidos, en estudiantes universitarios. Manuscrito sometido para publicación, Universidad de Concepción, Chile.

Molnar, B.E., Buka, S.L. \& Kessler, R.C. (2001). Child sexual abuse and subsequent psychopathology: Results from the national comorbidity survey. American Journal of Public Health, 91, 753-760.

Mullen, P.E., Martin, J.L., Anderson, J.C., Romans, S.E. \& Herbison, G.P. (1994) The effect of child sexual abuse on social, interpersonal, and sexual function in adult life. British Journal of Psychiatry, 165, $35-47$.

Mullen, P. E. \& Fleming, J. (1998). Long-term Effects of Child Sexual Abuse. Melbourne, Australian Institute of Family Studies. Issues in Child Abuse Prevention, 9. Recuperado el 20 de diciembre de 2008 desde http://www.aifs.gov.au/nch/pubs/issues/issues9/issues9.html\#rel

Najman, J. M., Dunne, M. P., Purdie, D.M., Boyle F.M. \& Coxeter, P. D. (2005). Sexual abuse in childhood and sexual dysfunction in adulthood: An Australian population-based study. Archives of Sexual Behavior, 34 (5), 517-526.

Oellerich, T.D. (2001). Child sexual abuse: Is the routine provision of psychotherapy warranted?. Issues in Children Abuse Acussations, 11 (1). Recuperado el 15 de Julio de 2008 desde http://www.ipt-forensics. com/journals.

Rempel, J. K., Holmes, J. G., \& Zanna, M. P. (1985). Trust in close relationship. Journal of Personality and Social Psychology, 49, 95-112.

Rind, B. \& Tromovitch, P. (1997). A meta-analytic review of findings from national samples on psychological correlates of child sexual abuse. The Journal of Sex Research, 34 (3), 237-255.

Rind, B., Tromovitch, P. \& Bauserman, R. (1998). A meta-analytic examination of assumed properties of child sexual abuse using collegue samples. Psychological Bulletin, 124 (1), 22-53.

Spataro, J., Mullen, P.E., Burgess, P.M., Wells, D.L. \& Moss, S.A. (2004). Impact of child sexual abuse on mental health. British Journal of Psychiatry, 184, 416-421

Swahnberg, I.M. K. \&Wijma, B. (2003). The NorVold Abuse Questionnaire (NorAQ). European Journal of Public Health, 13, 361-366.

Thelen, M.H., Vander Wal, J.S., Thomas, A.M. \& Harmon, R. (2000). Fear of intimacy among dating couples. Behavior Modification, 24 (2), 223-240. 